\title{
APPLICATIONS OF MEMBRANE COMPUTING
}




\section{Preface}

Membrane computing is a branch of natural computing which investigates computing models abstracted from the structure and functioning of living cells and from their interactions in tissues or higher order biological structures.

Briefly, a membrane system is a distributed computing model processing multisets of objects either in the compartments of a cell-like hierarchical arrangement of membranes (hence a structure of compartments which corresponds to a rooted tree), or in a tissue-like structure consisting of "cells" placed in the nodes of an arbitrary graph. Both the objects of the membranes, the membranes, and the links among them evolve according to some rules. For instance, the multisets of objects evolve mainly by means of rewriting rules, which have the form of usual chemical equations (several objects "react" and get transformed into some product objects). A crucial aspect of this processing is the resulting communication of objects through membranes, between regions of the same cell, between cells, or between cells and their environment.

A detailed introduction to membrane computing is provided in the first chapter of the book. This research area was initiated recently, at the end of 1998, with the aim of learning ideas, tools, techniques, and models from the biology of the cell that could turn out to be useful (or at least interesting) for the purpose of computing. The new field has flourished during the last five years; details can be found in the monograph Membrane Computing. An Introduction, published in 2002 in the same series as the present volume, and at the membrane systems (also called P systems) Website http://psystems . disco unimib.it). Many classes of P systems, inspired by either biological or mathematical considerations, were introduced, and their power (in comparison with various classes of Turing machines) and efficiency (the possibility to address/solve computationally hard problems) were extensively investigated.

Moreover, especially in recent years, it has turned out that membrane computing has significant potential to be applied to various problems of biology as well as to linguistics, theoretical computer science (sorting and ranking, 2D 
languages) and applied computer science (computer graphics, cryptography, approximate algorithms for optimization problems).

This book presents some applications of the membrane computing, organized in three main categories (biology, computer science, and linguistics), beginning with the introductory chapter mentioned above, and ending with a chapter about membrane computing software (useful in applications).

Before looking at these applications, let us discuss the attractiveness of membrane computing as a modeling framework, with an implicit reference to applications in biology.

First, there are several essential features genuinely relevant to membrane computing that are of interest for many applications:

- distribution (with important issues related to system-part interaction and emergent behavior nonlinearly resulting from the composition of local behaviors),

- discrete mathematics (continuous mathematics, especially systems of differential equations, has a glorious history of applications in many disciplines, such as astronomy, physics, and meteorology, but has failed to prove adequate for linguistics, and cannot cover more than local processes in biology because of the complexity of the processes and, in many cases, because of the imprecise character of the processes; a basic question is whether the biological reality is of continuous or discrete nature, as languages proved to be, with the latter ruling out the usefulness of many tools from continuous mathematics),

- algorithmicity (by definition, $\mathrm{P}$ systems are computability models of the same type as Turing machines or other classic representations of algorithms, and, as a consequence, they can be easily simulated on computers),

- scalability/extensibility (this is one of the main difficulties of using differential equations in biology),

- transparency (multiset rewriting rules are nothing other than reaction equations as customarily used in chemistry and biochemistry, without any "mysterious" notation or "mysterious" behavior),

- massive parallelism (a dream of computer science, a commonplace in biology),

- nondeterminism (let us compare the "program" of a P system, i.e., a set of rules localized in certain regions and without any imposed ordering, with the rigid sequences of instructions of programs written in typical programming languages),

- communication (with the astonishing and still not completely understood way in which life is coordinating the multitude of processes taking place in cells, tissues, organs, and organisms, in contrast with the costly way of coordinating/synchronizing computations in parallel electronic computing architectures, where the communication time becomes prohibitive with the increase in the number of processors). 
The majority of papers on applications of membrane computing are concerned with applications to biology. This is quite natural in view of the fact that the theory of membrane systems as a model of computation originated in biology as an abstraction of the structure and functioning of biological membranes. This supports our optimism about the (potential) success of these applications in modeling various aspects of biological reality, and should be contrasted with various models "transferred by force" from one area of science to another, where the original model was meant for totally different and incompatible phenomena (and consequently the modeling effort was unsuccessful).

Concerning applications reported till now, and hence applications reported in this volume, they are carried out at different levels. In many cases, what is actually used is the language of membrane computing, which involves (at least) three different aspects: (i) the long list of newly formulated concepts, (ii) the mathematical formalism of membrane computing, and (iii) the graphical way to represent membranes, cell-like structures, tissue-like structures, and so on.

Concerning graphical representation, we want to point out that not only the standard features (such as the hierarchical or tissue-like arrangements of membranes, the objects inhabiting the compartments, and the flow of information through communication channels), but also the evolution rules of the systems are part of the graphical representation. This makes visualization of the "evolution engine" transparent.

Another level of application is to use tools, techniques, and results obtained through research on membrane computing. These applications may aim at either

- solving problems already formulated by biologists, albeit informally or within a different model, or

- suggesting entirely new problems (problem areas) that become (more) transparent and interesting through the insights provided by the model of membrane computing.

Applications of all these types have been reported in the literature on membrane computing, and several of them are presented in this book. As already indicated, most applications concern biology. Moreover, applications to a number of other domains are also presented here. In particular, included are applications to computer graphics (where compartmentalization seems to add a significant efficiency to well known techniques based on L systems; see Chapter 9), linguistics (both as a representation language for various concepts related to language evolution, dialogue, semantics, Chapter 13, and making use of the parallelism for solving parsing problems in an efficient way, Chapter 14), management (again, mainly at the level of the formalism and the graphical language; such applications are not presented in the book, but references can be found on the Website mentioned above), sorting and ranking algorithms (Chapter 8), approximate algorithms for solving optimization problems 
(Chapter 11), cryptography (attacking a well known public-key cryptographic system; see Chapter 10), and NP-complete problems (the standard time-space trade-off; see Chapter 12).

The applications to biology in most cases follow a standard scenario. One examines a piece of reality, e.g., from the biochemistry of the cell, one writes a $\mathrm{P}$ system modeling the respective processes, one writes a program simulating this $\mathrm{P}$ system (or uses one of the existing programs for this purpose), and one performs a large number of experiments with the program (this is much cheaper than conducting laboratory experiments), tuning certain parameters and observing the evolution of the system (usually, following the population of certain objects). Illustrations of this strategy are given in Chapter 2 (modeling mechanosensitive channels), Chapter 4 (respiration in bacteria), Chapter 5 (cell-mediated immunity), Chapter 6 (photosynthesis), and Chapter 3 (gene expression regulation). Chapter 3 deserves special attention: it discusses a part of a more general research plan to develop a theory of discrete dynamical systems intimately related to both $\mathrm{P}$ systems and applications to biology.

Chapter 15 provides an overview of the available membrane computing software. Actually, these computer programs are not real "implementations" of $\mathrm{P}$ systems. This is due to the difficulties related to the nondeterminism and the parallelism; however, there are attempts to implement $\mathrm{P}$ systems on dedicated, reconfigurable hardware, as done by Petreska \& Teuscher, or on a cluster of computers, as done by Ciobanu \& Guo, or in a distributed fashion, as reported by Syropoulos et al. (references can be found in the bibliography of Chapter 15). At this moment, there is no bio-implementation of $\mathrm{P}$ systems.

Actually, as illustrated by the contributions to this volume, a lot remains to be done. It is the hope of the editors that this volume will contribute to and motivate future research in membrane computing. It is also our belief that membrane computing can offer a useful variety of tools, techniques, and models for a wide spectrum of applications.

We are much indebted to all contributors to this volume, admiring their patience and thanking them for their work during the many adjustments of the chapters. Special thanks are due to Springer-Verlag, in particular to Mrs. Ingeborg Mayer and Mr. Ronan Nugent, for very pleasant and efficient cooperation. 


\section{Contents}

1. Introduction to Membrane Computing

Gheorghe Păun

\section{Bio-Applications}

2. P System Models for Mechanosensitive Channels Ioan I. Ardelean, Daniela Besozzi, Max H. Garzon, Giancarlo Mauri, Sujoy Roy

3. P Systems for Biological Dynamics Luca Bianco, Federico Fontana, Giuditta Franco, Vincenzo Manca

4. Modeling Respiration in Bacteria and Respiration/Photosynthesis Interaction in Cyanobacteria Using a P System Simulator

Matteo Cavaliere, Ioan I. Ardelean

5. Modeling Cell-Mediated Immunity by Means of P Systems Gabriel Ciobanu

6. A Membrane Computing Model of Photosynthesis Taishin Yasunobu Nishida

7. Modeling p53 Signaling Pathways by Using Multiset Processing Yasuhiro Suzuki, Hiroshi Tanaka.

\section{Computer Science Applications}

8. Static Sorting P Systems

Artiom Alhazov, Dragoş Sburlan 
9. Membrane-Based Devices Used in Computer Graphics Alexandros Georgiou, Marian Gheorghe, Francesco Bernardini ....

10. An Analysis of a Public-Key Protocol with Membranes Olivier Michel, Florent Jacquemard

11. Membrane Algorithms: Approximate Algorithms for NP-Complete Optimization Problems Taishin Yasunobu Nishida

12. Computationally Hard Problems Addressed Through P Systems Mario J. Pérez-Jiménez, Alvaro Romero-Jiménez, Fernando Sancho-Caparrini

\section{Applications to Linguistics}

13. Linguistic Membrane Systems and Applications Gemma Bel Enguix, Maria Dolores Jiménez-Lopez

14. Parsing with P Automata

Radu Gramatovici, Gemma Bel Enguix

\section{Membrane Software}

15. Available Membrane Computing Software Miguel Angel Gutiérrez-Naranjo, Mario J. Pérez-Jiménez, Agustín Riscos-Núñez... 